\title{
STRONG STABILITY OF WEIGHTED SUMS OF NA RANDOM VARIABLES
}

\author{
GAN SHIXIN
}

Received 5 July 2003 and in revised form 1 April 2004

We study the almost sure (strong) stability of weighted sums of NA random variables and obtain some new results which extend earlier results of Matula (1992), Chow and Teicher (1971), Jamison et al. (1965), and Petrov (1975).

\section{Introduction and preliminaries}

We start with definitions. Let $(\Omega, \mathscr{F}, P)$ be a probability space. The random variables we deal with are all defined on $(\Omega, \mathscr{F}, P)$. A random variable sequence $\left\{Y_{n}, n \geq 1\right\}$ is said to be strongly stable if there exist two constant sequences $\left\{b_{n}\right\}$ and $\left\{d_{n}\right\}$ with $0<b_{n} \uparrow \infty$ such that

$$
b_{n}^{-1} Y_{n}-d_{n} \longrightarrow 0 \quad \text { a.s. }
$$

A random variable sequence $\left\{X_{n}, n \geq 1\right\}$ is said to be stochastically dominated by a nonnegative random variable $X$ (write $\left\{X_{n}\right\}<X$ ) if there exists a constant $c>0$ such that

$$
P\left(\left|X_{n}\right|>t\right) \leq c P(X>t) \quad \forall t>0, \forall n \geq 1 .
$$

A finite family of random variables $\left\{X_{i}, 1 \leq i \leq n\right\}$ is said to be negatively associated (abbreviated to NA) if for any disjoint subsets $A$ and $B$ of $\{1,2, \ldots, n\}$ and any real coordinatewise nondecreasing functions $f$ on $\mathbb{R}^{A}$ and $g$ on $\mathbb{R}^{B}$,

$$
\operatorname{Cov}\left(f\left(X_{i}, i \in A\right), g\left(X_{j}, j \in B\right)\right) \leq 0
$$

whenever the covariance exists. An infinite family of random variables $\left\{X_{i}, i \geq 1\right\}$ is NA if every finite subfamily is NA. This concept was introduced by Joag-Dev and Proschan [5]. They also pointed out and proved in their paper that a number of well-known multivariate distributions possess the NA property. Now people know that NA random variables have wide application in reliability theory and multivariate statistical analysis. Recently $\mathrm{Su}$ et al. [12] show that NA structure plays an important role in risk management. Because of these reasons, the notions of NA random variables have received more and more attention in recent years. A great number of papers for NA random variables have appeared in 
the literature. We refer to Joag-Dev and Proschab [5] for fundamental properties, Newmann [8] for the central limit theorem, Matula [7] for the three-series theorem, Shao and $\mathrm{Su}$ [11] for the law of the iterated logarithm, Shao [10] for moment inequalities, Liu et al. [6] for the Hàjek-Rènyi inequality, and Barbour et al. [1] for Poison approximation.

The main purpose of this paper is to study the strong stability of weighted sums of NA random variables and try to obtain some new results which extend the corresponding results of Matula [7], Chow and Teicher [2], Jamison et al. [4], and Petrov [9]. For this goal, we need some lemmas. The following lemma is a simple extension of [7, Theorem 3].

LEMmA 1.1. Let $\left\{X_{n}, n \geq 1\right\}$ be a sequence of NA random variables with finite second moments and $\left\{b_{n}, n \geq 1\right\}$ a sequence of real numbers with $0<b_{n} \uparrow \infty$. If $\sum_{n=1}^{\infty} \operatorname{Var}\left(X_{n}\right) / b_{n}^{2}<\infty$, then $\sum_{n=1}^{\infty}\left(X_{n}-E X_{n}\right) / b_{n}$ converges a.s., and therefore $\sum_{i=1}^{n}\left(X_{i}-E X_{i}\right) / b_{n} \rightarrow 0$ a.s.

Note that $\left\{X_{n} / b_{n}, n \geq 1\right\}$ is a sequence of NA random variables by [5, property $\left.\mathrm{P}_{6}\right]$. By using [7, Theorem 3], we can immediately obtain Lemma 1.1.

For a random variable $X$, write $X^{(c)}=X I(|X| \leq c)+c I(X>c)-c I(X<-c)$ for some $c>0$.

Lemma 1.2 (see [7, Theorem 4]). Let $\left\{X_{n}, n \geq 1\right\}$ be a sequence of NA random variables. If, for some $c>0$, the series $\sum_{n=1}^{\infty} E X_{n}^{(c)}, \sum_{n=1}^{\infty} \operatorname{Var}\left(X_{n}^{(c)}\right)$, and $\sum_{n=1}^{\infty} P\left(\left|X_{n}\right| \geq c\right)$ are convergent, then $\sum_{n=1}^{\infty} X_{n}$ is convergent a.s.

LEMma 1.3. Let $X$ be a random variable and $X_{0}$ a nonnegative random variable. If, for any $t>0, P(|X|>t) \leq c P\left(X_{0}>t\right)$, then for all $p>0, t>0$,

$$
E|X|^{p} I(|X| \leq t) \leq c\left(t^{p} P\left(X_{0}>t\right)+E X_{0}^{p} I\left(X_{0} \leq t\right)\right)
$$

Proof. By the integral equality

$$
p \int_{0}^{t} s^{p-1} P(|X|>s) d s=t^{p} P(|X|>t)+E|X|^{p} I(|X| \leq t)
$$

it follows that

$$
\begin{aligned}
E|X|^{p} I(|X| \leq t) & \leq p \int_{0}^{t} s^{p-1} P(|X|>s) d s \leq c p \int_{0}^{t} s^{p-1} P\left(X_{0}>s\right) d s \\
& =c\left(t^{p} P\left(X_{0}>t\right)+E X_{0}^{p} I\left(X_{0} \leq t\right)\right) .
\end{aligned}
$$

LEMMA 1.4. Let $\left\{a_{n}, n \geq 1\right\}$ and $\left\{b_{n}, n \geq 1\right\}$ be two sequences of positive numbers with $c_{n}=$ $b_{n} / a_{n}$ and $b_{n} \uparrow \infty$. Let $\left\{X_{n}, n \geq 1\right\}$ be a sequence of mean zero variables with $\left\{X_{n}\right\}<X$, where $X$ is a nonnegative random variable. Define $N(x)=\operatorname{Card}\left\{n: c_{n} \leq x\right\}, x>0$. If

(1) $\sum_{i=1}^{\infty} P\left(\left|X_{i}\right|>c_{i}\right)<\infty$,

(2) $\sum_{n=1}^{\infty} \int_{1}^{\infty} P\left(\left|X_{n}\right|>s c_{n}\right) d s<\infty$, or

(1') $E N(X)<\infty$,

$\left(2^{\prime}\right) \int_{1}^{\infty} E N(X / s)<\infty$, then

$$
b_{n}^{-1} \sum_{i=1}^{n} a_{i} E X_{i}^{\left(c_{i}\right)} \longrightarrow 0 \quad \text { as } n \longrightarrow \infty .
$$


Proof. Obviously $\left(1^{\prime}\right) \Rightarrow(1)$ and $\left(2^{\prime}\right) \Rightarrow(2)$. It suffices to show that under conditions (1) and (2) we have (1.7):

$$
\begin{aligned}
\sum_{i=1}^{\infty} \frac{a_{i}\left|E X_{i}^{\left(c_{i}\right)}\right|}{b_{i}} & =\sum_{i=1}^{\infty} c_{i}^{-1}\left|E\left(X_{i}-c_{i}\right) I\left(X_{i}>c_{i}\right)+E\left(X_{i}+c_{i}\right) I\left(X_{i}<-c_{i}\right)\right| \\
& \leq \sum_{i=1}^{\infty} c_{i}^{-1} E\left(\left|X_{i}\right|+c_{i}\right) I\left(\left|X_{i}\right|>c_{i}\right) \\
& =\sum_{i=1}^{\infty} c_{i}^{-1} E\left|X_{i}\right| I\left(\left|X_{i}\right|>c_{i}\right)+\sum_{i=1}^{\infty} P\left(\left|X_{i}\right|>c_{i}\right), \\
\sum_{i=1}^{\infty} c_{i}^{-1} E\left|X_{i}\right| I\left(\left|X_{i}\right|>c_{i}\right) & \leq \sum_{i=1}^{\infty} c_{i}^{-1}\left(c_{i} P\left(\left|X_{i}\right|>c_{i}\right)+\int_{c_{i}}^{\infty} P\left(\left|X_{i}\right|>t\right) d t\right) \\
& =\sum_{i=1}^{\infty} P\left(\left|X_{i}\right|>c_{i}\right)+\sum_{i=1}^{\infty} \int_{1}^{\infty} P\left(\left|X_{i}\right|>s c_{i}\right) d s<\infty .
\end{aligned}
$$

Therefore, from (1.8) and (1.9), $\sum_{i=1}^{\infty} a_{i} E X_{i}^{\left(c_{i}\right)} / b_{i}$ converges. By Kronecker's lemma, we have (1.7).

Corollary 1.5. Let $\left\{X_{n}, n \geq 1\right\}$ be a sequence of mean zero random variables with $\left\{X_{n}\right\}<$ $X \in L \log ^{+} L$. If

(1) $\sum_{n=1}^{\infty} P\left(\left|X_{n}\right|>n\right)<\infty$,

(2) $\sum_{n=1}^{\infty} \int_{1}^{\infty} P\left(\left|X_{n}\right|>s n\right) d s<\infty$, then

$$
n^{-1} \sum_{i=1}^{n} E X_{i}^{(i)} \longrightarrow 0 \text { as } n \longrightarrow \infty .
$$

Proof. Clearly $c_{n}=n, n \geq 1$. The first part of proof is like in Lemma 1.4. We only give the last part of proof:

$$
\begin{aligned}
& \sum_{i=1}^{\infty} i^{-1} E\left|X_{i}\right| I\left(\left|X_{i}\right|>i\right) \\
& \quad \leq \sum_{i=1}^{\infty} i^{-1}\left[i P\left(\left|X_{i}\right|>i\right)+\int_{i}^{\infty} P\left(\left|X_{i}\right|>t\right) d t\right] \\
& \leq c \sum_{i=1}^{\infty} P(X>i)+c \sum_{i=1}^{\infty} i^{-1} \int_{i}^{\infty} P(X>t) d t \\
& \leq c E X+c \sum_{i=1}^{\infty} i^{-1} \sum_{k=i}^{\infty} P(X>k) \leq c E X+c \sum_{k=1}^{\infty} \sum_{i=1}^{k} i^{-1} P(X>k) \\
& \leq c E X+c \sum_{k=1}^{\infty}(1+\log k) P(X>k) \leq c E X+c E X \log ^{+} X<\infty .
\end{aligned}
$$

This shows that $n^{-1} \sum_{i=1}^{n} E X_{i}^{(i)} \rightarrow 0$ as $n \rightarrow \infty$. 
978 Strong stability of weighted sums of NA random variables

Throughout this paper, the symbol $c$ stands for a generic positive constant which may differ from one place to another.

\section{Strong stability}

Theorem 2.1. Let $\left\{X_{n}, n \geq 1\right\}$ be a sequence of $N A$ random variables and $\left\{g_{n}(x), n \geq 1\right\}$ a sequence of even functions, positive and nondecreasing in the interval $x>0$. If one of the following conditions is satisfied for every $n \geq 1$ :

(1) $x / g_{n}(x) \uparrow$ as $0<x \uparrow$,

(2) $x / g_{n}(x) \downarrow$ and $g_{n}(x) / x^{2} \uparrow$ as $0<x \uparrow$ and also $E X_{n}=0$,

then for any positive real-number sequence $\left\{b_{n}, n \geq 1\right\}$ with $b_{n} \uparrow \infty$ satisfying

$$
\sum_{n=1}^{\infty} \frac{E g_{n}\left(X_{n}\right)}{g_{n}\left(b_{n}\right)}<\infty
$$

the series $\sum_{n=1}^{\infty} X_{n} / b_{n}$ converges almost surely, and therefore $\sum_{i=1}^{n} X_{i} / b_{n} \rightarrow 0$ a.s.

Proof. For each $n \geq 1$, put $Z_{n}=X_{n} / b_{n}$. Then $\left\{Z_{n}, n \geq 1\right\}$ remains a sequence of NA random variables by $\left[5\right.$, property $\left.\mathrm{P}_{6}\right]$. Take $c=1$ in Lemma 1.2. Since $g_{n}(x) \uparrow$ as $x>0$, then $g_{n}\left(\left|X_{n}\right|\right) \geq g_{n}\left(b_{n}\right)$ on $\left\{\left|Z_{n}\right| \geq 1\right\}$. So

$$
\sum_{n=1}^{\infty} P\left(\left|Z_{n}\right| \geq 1\right) \leq \sum_{n=1}^{\infty} \int \frac{g_{n}\left(\left|X_{n}\right|\right) I\left(\left|Z_{n}\right| \geq 1\right)}{g_{n}\left(b_{n}\right)} d P \leq \sum_{n=1}^{\infty} \frac{E g_{n}\left(X_{n}\right)}{g_{n}\left(b_{n}\right)}<\infty .
$$

We will suppose that the function $g_{n}(x)$ satisfies condition (1), then, in the interval $|x| \leq$ $b_{n}$, we have $x^{2} / b_{n}^{2} \leq g_{n}^{2}(x) / g_{n}^{2}\left(b_{n}\right) \leq g_{n}(x) / g_{n}\left(b_{n}\right)$. If, however, $n$ is such that (2) is satisfied, then, in the same interval, we have $x^{2} / b_{n}^{2} \leq g_{n}(x) / g_{n}\left(b_{n}\right)$, therefore

$$
\begin{aligned}
\sum_{n=1}^{\infty} E\left(Z_{n}^{(1)}\right)^{2} & =\sum_{n=1}^{\infty}\left(\int Z_{n}^{2} I\left(\left|Z_{n}\right| \leq 1\right) d P+\int I\left(\left|Z_{n}\right|>1\right) d P\right) \\
& \leq \sum_{n=1}^{\infty}\left(\int \frac{g_{n}\left(X_{n}\right) I\left(\left|X_{n}\right| \leq b_{n}\right)}{g_{n}\left(b_{n}\right)} d P+\int \frac{g_{n}\left(X_{n}\right) I\left(\left|X_{n}\right|>b_{n}\right)}{g_{n}\left(b_{n}\right)} d P\right) \\
& =\sum_{n=1}^{\infty} \frac{E g_{n}\left(X_{n}\right)}{g_{n}\left(b_{n}\right)}<\infty .
\end{aligned}
$$

If condition (1) is satisfied, then

$$
\begin{aligned}
\left|E Z_{n}^{(1)}\right| & \leq \int \frac{\left|X_{n}\right| I\left(\left|X_{n}\right| \leq b_{n}\right)}{b_{n}} d P+\int I\left(\left|X_{n}\right|>b_{n}\right) d P \\
& \leq \int \frac{g_{n}\left(\left|X_{n}\right|\right) I\left(\left|X_{n}\right| \leq b_{n}\right)}{g_{n}\left(b_{n}\right)} d P+\int \frac{g_{n}\left(\left|X_{n}\right|\right) I\left(\left|X_{n}\right|>b_{n}\right)}{g_{n}\left(b_{n}\right)} d P \\
& =\frac{E g_{n}\left(X_{n}\right)}{g_{n}\left(b_{n}\right)} .
\end{aligned}
$$


If condition (2) is satisfied, then

$$
\begin{aligned}
\left|E Z_{n}^{(1)}\right| & =\left|\int\left(Z_{n}-1\right) I\left(Z_{n}>1\right) d P+\int\left(Z_{n}+1\right) I\left(Z_{n}<-1\right) d P\right| \\
& \leq \int\left|Z_{n}\right| I\left(\left|Z_{n}\right|>1\right) d P+\int I\left(\left|Z_{n}\right|>1\right) d P \\
& \leq \int \frac{g_{n}\left(\left|X_{n}\right|\right) I\left(\left|Z_{n}\right|>1\right)}{g_{n}\left(b_{n}\right)} d P+\int \frac{g_{n}\left(\left|X_{n}\right|\right) I\left(\left|Z_{n}\right|>1\right)}{g_{n}\left(b_{n}\right)} d P \\
& \leq \frac{2 E g_{n}\left(X_{n}\right)}{g_{n}\left(b_{n}\right)} .
\end{aligned}
$$

Consequently, we have

$$
\sum_{n=1}^{\infty}\left|E Z_{n}^{(1)}\right| \leq 2 \sum_{n=1}^{\infty} \frac{E g_{n}\left(X_{n}\right)}{g_{n}\left(b_{n}\right)}<\infty
$$

Thus (2.2), (2.3), (2.6), and Lemma 1.2 imply the convergence of $\sum_{n=1}^{\infty} Z_{n}=\sum_{n=1}^{\infty} X_{n} / b_{n}$ almost surely.

Take $g_{n}(x)=|x|^{p}, n \geq 1,1 \leq p \leq 2$, in Theorem 2.1. We can infer the following important special case which is a further extension of Lemma 1.1.

Corollary 2.2. Let $\left\{X_{n}, n \geq 1\right\}$ be a sequence of NA mean zero random variables and $\left\{b_{n}, n \geq 1\right\}$ a sequence of real numbers with $0<b_{n} \uparrow \infty, 1 \leq p \leq 2$. If $\sum_{n=1}^{\infty} E\left|X_{n}\right| p / b_{n}^{p}<\infty$, then $\sum_{i=1}^{n} X_{i} / b_{n} \rightarrow 0$ a.s.

Theorem 2.3. Let $\left\{a_{n}, n \geq 1\right\}$ and $\left\{b_{n}, n \geq 1\right\}$ be two sequences of positive numbers with $c_{n}=b_{n} / a_{n}$ and $b_{n} \uparrow \infty$. Let $\left\{X_{n}, n \geq 1\right\}$ be a sequence of $N A$ random variables which is stochastically dominated by a nonnegative random variable $X$. Set $N(x)=\operatorname{Card}\left\{n: c_{n} \leq x\right\}$, $x>0.1 \leq p \leq 2$. If the following conditions are satisfied:

(1) $E N(X)<\infty$,

(2) $\int_{0}^{\infty} t^{p-1} P(X>t) \int_{t}^{\infty} N(y) / y^{p+1} d y d t<\infty$,

then there exist $d_{n} \in \mathbb{R}, n=1,2, \ldots$ such that

$$
b_{n}^{-1} \sum_{i=1}^{n} a_{i} X_{i}-d_{n} \longrightarrow 0 \quad \text { a.s. }
$$

Proof. Let $S_{n}=\sum_{i=1}^{n} a_{i} X_{i}, T_{n}=\sum_{i=1}^{n} a_{i} X_{i}^{\left(c_{i}\right)}, n \geq 1$. Obviously we have

$$
\sum_{i=1}^{\infty} P\left(X_{i} \neq X_{i}^{\left(c_{i}\right)}\right)=\sum_{i=1}^{\infty} P\left(\left|X_{i}\right|>c_{i}\right) \leq c \sum_{i=1}^{\infty} P\left(X>c_{i}\right) \leq c E N(X)<\infty .
$$

By Borel-Cantelli lemma for any sequence $\left\{d_{n}\right\} \subset \mathbb{R}$, the sequences $\left\{b_{n}^{-1} T_{n}-d_{n}\right\}$ and $\left\{b_{n}^{-1} S_{n}-d_{n}\right\}$ converge on the same set and to the same limit. We will show that $b_{n}^{-1} \sum_{i=1}^{n} a_{i}$ $\left(X_{i}^{\left(c_{i}\right)}-E X_{i}^{\left(c_{i}\right)}\right) \rightarrow 0$ a.s. which gives the theorem with $d_{n}=b_{n}^{-1} \sum_{i=1}^{n} a_{i} E X_{i}^{\left(c_{i}\right)}$. Now note that $\left\{a_{i}\left(X_{i}^{\left(c_{i}\right)}-E X_{i}^{\left(c_{i}\right)}\right), i \geq 1\right\}$ is a sequence of NA mean zero random variables by 
980 Strong stability of weighted sums of NA random variables

$\left[5\right.$, property $\left.\mathrm{P}_{6}\right]$. It follows from $c_{r}$-inequality and Lemma 1.3 that

$$
\begin{aligned}
& \sum_{n=1}^{\infty} \frac{E\left|a_{n}\left(X_{n}^{\left(c_{n}\right)}-E X_{n}^{\left(c_{n}\right)}\right)\right|^{p}}{b_{n}^{p}} \\
& \quad \leq c \sum_{n=1}^{\infty} c_{n}^{-p} E\left|X_{n}\right|^{p} I\left(\left|X_{n}\right| \leq c_{n}\right)+c \sum_{n=1}^{\infty} P\left(\left|X_{n}\right|>c_{n}\right) \\
& \quad \leq c \sum_{n=1}^{\infty} c_{n}^{-p}\left(c_{n}^{p} P\left(X>c_{n}\right)+E X^{p} I\left(X \leq c_{n}\right)\right)+c \sum_{n=1}^{\infty} P\left(X>c_{n}\right) \\
& \quad \leq c E N(X)+c \sum_{n=1}^{\infty} p c_{n}^{-p} \int_{0}^{c_{n}} t^{p-1} P(X>t) d t, \\
& \sum_{n=1}^{\infty} p c_{n}^{-p} \int_{0}^{c_{n}} t^{p-1} P(X>t) d t=p \int_{0}^{\infty} t^{p-1} P(X>t) \sum_{\left\{n: c_{n}>t\right\}} c_{n}^{-p} d t \\
& \leq p^{2} \int_{0}^{\infty} t^{p-1} P(X>t) \int_{t}^{\infty} \frac{N(y)}{y^{p+1}} d y d t .
\end{aligned}
$$

The last inequality follows from the fact that

$$
\begin{aligned}
\sum_{\left\{n: c_{n}>t\right\}} c_{n}^{-p} & =\lim _{u \rightarrow \infty} \sum_{\left\{n: t<c_{n}<u\right\}} c_{n}^{-p}=\lim _{u \rightarrow \infty} \int_{t}^{u} y^{-p} d N(y) \\
& =\lim _{u \rightarrow \infty}\left(u^{-p} N(u)-t^{-p} N(t)+\int_{t<y \leq u} y^{-(p+1)} N(y) d y\right)
\end{aligned}
$$

and $u^{-p} N(u) \leq p \int_{u}^{\infty} y^{-(p+1)} N(y) d y \rightarrow 0$ as $u \rightarrow \infty$. Hence, $\sum_{n=1}^{\infty}\left|a_{n}\left(X_{n}^{\left(c_{n}\right)}-E X_{n}^{\left(c_{n}\right)}\right)\right|^{p /}$ $b_{n}^{p}<\infty$. By Corollary 2.2, it follows that $b_{n}^{-1} \sum_{i=1}^{n} a_{i}\left(X_{i}^{\left(c_{i}\right)}-E X_{i}^{\left(c_{i}\right)}\right) \rightarrow 0$ a.s. This completes the proof of Theorem 2.3.

Remark 2.4. Heyde's [3, Theorem 2] extended [4, Theorem 2] of Jamsion et al. to more general weights, which are just the same weights considered in our paper. So Theorem 2.3 is an extension of [3, Theorem 2].

From Theorem 2.3 and Lemma 1.4, we have the following corollaries.

Corollary 2.5. Let the conditions of Theorem 2.3 be fulfilled and $E X_{n}=0, n \geq 1$, and $\int_{1}^{\infty} E N(X / s) d s<\infty$. Then $b_{n}^{-1} \sum_{i=1}^{n} a_{i} X_{i} \rightarrow 0$ a.s.

Corollary 2.6. Let $\left\{X_{n}, n \geq 1\right\}$ be a sequence of NA mean zero random variables and $\left\{X_{n}\right\}<X$.

(1) If $X \in L \log ^{+} L$, then $n^{-1} \sum_{i=1}^{n} X_{i} \rightarrow 0$ a.s.

(2) If $X \in L^{r}, 1<r<2$, then $n^{-1 / r} \sum_{i=1}^{n} X_{i} \rightarrow 0$ a.s. 
Proof. (1) Take $a_{n}=1, b_{n}=n, n \geq 1, p>1$, in Theorem 2.3, then $c_{n}=n, N(x)=\operatorname{Card}\{n$ : $\left.c_{n} \leq x\right\} \leq x, x \geq 0$. It is easy to show that conditions (1) and (2) in Theorem 2.3 hold true. Theorem 2.3 and Corollary 1.5 guarantee that $n^{-1} \sum_{i=1}^{n} X_{i} \rightarrow 0$ a.s.

(2) Take $a_{n}=1, b_{n}=n^{1 / r}, n \geq 1, p=2$, in Theorem 2.3. Put $\delta=2-r>0$, then $N(x)=$ Card $\left\{n: n^{1 / r} \leq x\right\} \leq x^{r}, x \geq 0$. It is easy to verify that the conditions in Corollary 2.5 hold true. By Corollary 2.5, we have $n^{-1 / r} \sum_{i=1}^{n} X_{i} \rightarrow 0$ a.s.

Theorem 2.7. Let $\left\{a_{n}, n \geq 1\right\}$ and $\left\{b_{n}, n \geq 1\right\}$ be two sequences of positive numbers with $c_{n}=b_{n} / a_{n}$ and $b_{n} \uparrow \infty$. Let $\left\{X_{n}, n \geq 1\right\}$ be a sequence of $N A$ random variables which is stochastically dominated by a nonnegative random variable $X . N(x)=\operatorname{Card}\left\{n: c_{n} \leq x\right\}$, $x>0.1 \leq p \leq 2$. If the following conditions are satisfied:

(1) $E N(X)<\infty$,

(2) $\int_{1}^{\infty} E N(X / s) d s<\infty$,

(3) $\max _{1 \leq j \leq n} c_{j}^{p} \sum_{j=n}^{\infty} c_{j}^{-p}=O(n)$,

then

$$
b_{n}^{-1} \sum_{i=1}^{n} a_{i} X_{i} \longrightarrow 0 \quad \text { a.s. }
$$

Proof.

$$
\sum_{n=1}^{\infty} P\left(X_{n} \neq X_{n}^{\left(c_{n}\right)}\right)=\sum_{n=1}^{\infty} P\left(\left|X_{n}\right|>c_{n}\right) \leq c \sum_{n=1}^{\infty} P\left(X>c_{n}\right) \leq c E N(X)<\infty
$$

By Borel-Cantelli lemma, it suffices to show that $b_{n}^{-1} \sum_{i=1}^{n} a_{i} X_{i}^{\left(c_{i}\right)} \rightarrow 0$ a.s. From Lemma 1.4 , we need only to show that $b_{n}^{-1} \sum_{i=1}^{n} a_{i}\left(X_{i}^{\left(c_{i}\right)}-E X_{i}^{\left(c_{i}\right)}\right) \rightarrow 0$ a.s. Note that $\left\{a_{i}\left(X_{i}^{\left(c_{i}\right)}-\right.\right.$ $\left.\left.E X_{i}^{\left(c_{i}\right)}\right), i \geq 1\right\}$ is a sequence of NA mean zero random variables.

$$
\begin{aligned}
& \sum_{n=1}^{\infty} \frac{E\left|a_{n}\left(X_{n}^{\left(c_{n}\right)}-E X_{n}^{\left(c_{n}\right)}\right)\right|^{p}}{b_{n}^{p}} \\
& \quad \leq c \sum_{n=1}^{\infty} c_{n}^{-p} E\left|X_{n}^{\left(c_{n}\right)}\right|^{p}=c \sum_{n=1}^{\infty} c_{n}^{-p}\left(E\left|X_{n}\right|^{p} I\left(\left|X_{n}\right| \leq c_{n}\right)+c_{n}^{p} P\left(\left|X_{n}\right|>c_{n}\right)\right) \\
& \quad \leq c \sum_{n=1}^{\infty} c_{n}^{-p}\left(c_{n}^{p} P\left(X>c_{n}\right)+E X^{p} I\left(X \leq c_{n}\right)\right)+c \sum_{n=1}^{\infty} P\left(X>c_{n}\right) \\
& \quad \leq c \sum_{n=1}^{\infty} P\left(X>c_{n}\right)+c \sum_{n=1}^{\infty} \frac{E X^{p} I\left(X \leq c_{n}\right)}{c_{n}^{p}}
\end{aligned}
$$

Clearly

$$
\sum_{n=1}^{\infty} P\left(X>c_{n}\right) \leq E N(X)<\infty
$$


982 Strong stability of weighted sums of NA random variables

Put $\varepsilon_{n}=\max _{1 \leq j \leq n} c_{j}, \varepsilon_{0}=0$, then

$$
\begin{aligned}
& \sum_{n=1}^{\infty} \frac{E X^{p} I\left(X \leq c_{n}\right)}{c_{n}^{p}} \leq \sum_{n=1}^{\infty} \frac{E X^{p} I\left(X \leq \varepsilon_{n}\right)}{c_{n}^{p}}=\sum_{n=1}^{\infty} \sum_{j=1}^{n} \frac{E X^{p} I\left(\varepsilon_{j-1}<X \leq \varepsilon_{j}\right)}{c_{n}^{p}} \\
& \leq \sum_{j=1}^{\infty} P\left(\varepsilon_{j-1}<X \leq \varepsilon_{j}\right) \varepsilon_{j}^{p} \sum_{n=j}^{\infty} \frac{1}{c_{n}^{p}} \leq c \sum_{j=1}^{\infty} j P\left(\varepsilon_{j-1}<X \leq \varepsilon_{j}\right) \\
& =c \sum_{j=1}^{\infty} \sum_{n=1}^{j} P\left(\varepsilon_{j-1}<X \leq \varepsilon_{j}\right)=c \sum_{n=1}^{\infty} P\left(X>\varepsilon_{n-1}\right) \\
& \leq c\left(1+\sum_{n=1}^{\infty} P\left(X>c_{n}\right)\right)<\infty \text {. }
\end{aligned}
$$

By Corollary 2.2, we have $b_{n}^{-1} \sum_{i=1}^{n} a_{i}\left(X_{i}^{\left(c_{i}\right)}-E X_{i}^{\left(c_{i}\right)}\right) \rightarrow 0$ a.s. The proof is complete.

Afterwards let $\alpha(x): \mathbb{R}_{+} \rightarrow \mathbb{R}_{+}$be a positive, nonincreasing function with $a_{n}=\alpha(n)$, $b_{n}=\sum_{i=1}^{n} a_{i}, c_{n}=b_{n} / a_{n}, n \geq 1$, where

$$
\begin{gathered}
b_{n} \longrightarrow \infty, \\
0<\liminf _{n \rightarrow \infty} n^{-1} c_{n} \alpha\left(\log c_{n}\right) \leq \limsup _{n \rightarrow \infty} n^{-1} c_{n} \alpha\left(\log c_{n}\right)<\infty, \\
x \alpha\left(\log ^{+} x\right) \text { is nondecreasing for } x>0 .
\end{gathered}
$$

TheOREm 2.8. Let $\left\{X_{n}, n \geq 1\right\}$ be a sequence of identically distributed NA random variables. If $E\left|X_{1}\right| \alpha\left(\log ^{+}\left|X_{1}\right|\right)<\infty$, then there exist $d_{n} \in \mathbb{R}, n=1,2, \ldots$, such that $b_{n}^{-1} \sum_{i=1}^{n} a_{i} X_{i}-$ $d_{n} \rightarrow 0$ a.s.

Proof. Since $0<\alpha(x) \downarrow, b_{n} \uparrow \infty$, then $c_{n} \uparrow \infty$. By (2.18) we can choose $m_{0} \in \mathbb{N}, \gamma>0, \beta>0$, such that, for $n \geq m_{0}$,

$$
\gamma n \leq c_{n} \alpha\left(\log c_{n}\right) \leq \beta n
$$

Hence, for $n \geq m \geq m_{0}$, we have $c_{n} \geq \gamma n\left(\alpha\left(\log c_{m}\right)\right)^{-1}$ which guarantees that

$$
\sum_{j=m}^{\infty} c_{j}^{-2} \leq \frac{\alpha^{2}\left(\log c_{m}\right)}{\gamma^{2} m}, \quad m \geq m_{0}
$$

Note that $\left\{X_{i}^{\left(c_{i}\right)}, i \geq 1\right\}$ is a sequence of NA random variables by [5, property $\mathrm{P}_{6}$ ]. For $m \geq m_{0}$,

$$
\sum_{j=m}^{\infty} \frac{E\left(X_{j}^{\left(c_{j}\right)}\right)^{2}}{c_{j}^{2}}=\sum_{j=m}^{\infty} c_{j}^{-2} E X_{j}^{2} I\left(\left|X_{j}\right| \leq c_{j}\right)+\sum_{j=m}^{\infty} P\left(\left|X_{j}\right|>c_{j}\right) .
$$


From (2.20) and (2.21), it follows that, for $m \geq m_{0}$,

$$
\begin{aligned}
\sum_{j=m}^{\infty} P\left(\left|X_{j}\right|>c_{j}\right) & \leq \sum_{j=m}^{\infty} P\left(\left|X_{j}\right| \alpha\left(\log ^{+}\left|X_{j}\right|\right) \geq c_{j} \alpha\left(\log c_{j}\right)\right) \\
& \leq \sum_{j=m}^{\infty} P\left(\left|X_{j}\right| \alpha\left(\log ^{+}\left|X_{j}\right|\right) \geq \gamma j\right) \\
& =\sum_{j=m}^{\infty} P\left(\left|X_{1}\right| \alpha\left(\log ^{+}\left|X_{1}\right|\right) \geq \gamma j\right)<\infty \\
\sum_{j=m}^{\infty} c_{j}^{-2} E X_{j}^{2} I\left(\left|X_{j}\right| \leq c_{j}\right) & =\sum_{j=m}^{\infty} c_{j}^{-2} E X_{1}^{2} I\left(\left|X_{1}\right| \leq c_{j}\right) \\
& \leq \sum_{j=m}^{\infty} c_{j}^{-2}\left(\int_{\left\{\left|X_{1}\right| \leq c_{m-1}\right\}} X_{1}^{2} d P+\sum_{i=m}^{j} \int_{\left\{c_{i-1}<\left|X_{1}\right| \leq c_{i}\right\}} X_{1}^{2} d P\right) \\
& \leq O(1)+\sum_{j=m}^{\infty} c_{j}^{-2} \sum_{i=m}^{j} \int_{\left\{c_{i-1}<\left|X_{1}\right| \leq c_{i}\right\}} X_{1}^{2} d P \\
& \leq O(1)+\sum_{i=m}^{\infty} \gamma^{-2} i^{-1} \alpha^{2}\left(\log c_{i}\right) \int_{\left\{c_{i-1}<\left|X_{1}\right| \leq c_{i}\right\}} X_{1}^{2} d P \\
& \leq O(1)+\beta \gamma^{-2} \sum_{i=m}^{\infty} \alpha\left(\log c_{i}\right) \int_{\left\{c_{i-1}<\left|X_{1}\right| \leq c_{i}\right\}}\left|X_{1}\right| d P \\
& O X_{1} \mid \alpha\left(\log { }^{+}\left|X_{1}\right|\right) d P<\infty .
\end{aligned}
$$

From (2.22)-(2.24), it follows that

$$
\sum_{j=1}^{\infty} \frac{\operatorname{Var}\left(X_{j}^{\left(c_{j}\right)}\right)}{c_{j}^{2}} \leq \sum_{j=1}^{\infty} \frac{E\left(X_{j}^{\left(c_{j}\right)}\right)^{2}}{c_{j}^{2}}<\infty .
$$

By Lemma 1.1, we have $b_{n}^{-1} \sum_{i=1}^{n} a_{i}\left(X_{i}^{\left(c_{i}\right)}-E X_{i}^{\left(c_{i}\right)}\right) \rightarrow 0$ a.s. On the other hand, by (2.23), we have

$$
\begin{aligned}
\sum_{i=1}^{\infty} P\left(X_{i} \neq X_{i}^{\left(c_{i}\right)}\right) & =\sum_{i=1}^{\infty} P\left(\left|X_{i}\right|>c_{i}\right)=\sum_{i=1}^{m_{0}-1} P\left(\left|X_{i}\right|>c_{i}\right)+\sum_{i=m_{0}}^{\infty} P\left(\left|X_{i}\right|>c_{i}\right) \\
& \leq m_{0}-1+\sum_{i=m_{0}}^{\infty} P\left(\left|X_{1}\right|>c_{i}\right)<\infty
\end{aligned}
$$

Take $d_{n}=b_{n}^{-1} \sum_{i=1}^{n} a_{i} E X_{i}^{\left(c_{i}\right)}, n \geq 1$. By Borel-Cantelli Lemma, we know that the theorem holds true. 
984 Strong stability of weighted sums of NA random variables

If $\left\{X_{n}, n \geq 1\right\}$ is not identically distributed, we have the following result.

Theorem 2.9. Let $\left\{X_{n}, n \geq 1\right\}$ be a sequence of $N A$ random variables (not necessarily identically distributed). $1 \leq p \leq 2$. If $\sum_{n=1}^{\infty} n^{-p} E\left|X_{n} \alpha\left(\log ^{+}\left|X_{n}\right|\right)\right|^{p}<\infty$, then there exist $\left\{d_{n}\right\} \subset \mathbb{R}$ such that $b_{n}^{-1} \sum_{i=1}^{n} a_{i} X_{i}-d_{n} \rightarrow 0$ a.s.

Proof. Using the same notations and similar method of Theorem 2.8, we can easily get

$$
\begin{aligned}
\sum_{i=1}^{\infty} P\left(X_{i} \neq X_{i}^{\left(c_{i}\right)}\right) & \leq m_{0}-1+\sum_{i=m_{0}}^{\infty} P\left(\left|X_{i}\right|>c_{i}\right) \\
& \leq m_{0}-1+\sum_{i=m_{0}}^{\infty} P\left(\left|X_{i} \alpha\left(\log ^{+}\left|X_{i}\right|\right)\right|>c_{i} \alpha\left(\log c_{i}\right)\right) \\
& \leq m_{0}-1+\sum_{i=m_{0}}^{\infty} P\left(\left|X_{i} \alpha\left(\log ^{+}\left|X_{i}\right|\right)\right| \geq \gamma i\right) \\
& \leq m_{0}-1+\gamma^{-p} \sum_{i=m_{0}}^{\infty} i^{-p} E\left|X_{i} \alpha\left(\log ^{+}\left|X_{i}\right|\right)\right|^{p}<\infty .
\end{aligned}
$$

By Borel-Cantelli lemma for any sequence $\left\{d_{n}\right\} \subset \mathbb{R}$, the sequences $\left\{b_{n}^{-1} \sum_{i=1}^{n} a_{i} X_{i}^{\left(c_{i}\right)}-d_{n}\right\}$ and $\left\{b_{n}^{-1} \sum_{i=1}^{n} a_{i} X_{i}-d_{n}\right\}$ converge on the same set and to the same limit. We will show that $b_{n}^{-1} \sum_{i=1}^{n} a_{i}\left(X_{i}^{\left(c_{i}\right)}-E X_{i}^{\left(c_{i}\right)}\right) \rightarrow 0$ a.s. which gives the theorem with $d_{n}=b_{n}^{-1} \sum_{i=1}^{n} a_{i} E X_{i}^{\left(c_{i}\right)}$, $n \geq 1$. Now note that $\left\{a_{i}\left(X_{i}^{\left(c_{i}\right)}-E X_{i}^{\left(c_{i}\right)}\right), i \geq 1\right\}$ is a sequence of NA mean zero random variables.

$$
\begin{aligned}
& \sum_{n=1}^{\infty} b_{n}^{-p} E\left|a_{n}\left(X_{n}^{\left(c_{n}\right)}-E X_{n}^{\left(c_{n}\right)}\right)\right|^{p} \\
& \quad \leq c \sum_{n=1}^{\infty} c_{n}^{-p} E\left|X_{n}^{\left(c_{n}\right)}\right|^{p} \\
& \quad \leq c\left(m_{0}-1\right)+c \sum_{n=m_{0}}^{\infty} c_{n}^{-p}\left(E\left|X_{n}\right|^{p} I\left(\left|X_{n}\right| \leq c_{n}\right)+c_{n}^{p} P\left(\left|X_{n}\right|>c_{n}\right)\right) \\
& \quad \leq c\left(m_{0}-1\right)+c \sum_{n=m_{0}}^{\infty} c_{n}^{-p}\left(E\left|X_{n}\right|{ }^{p} I\left(\left|X_{n}\right| \leq c_{n}\right)+c \sum_{n=m_{0}}^{\infty} P\left(\left|X_{n}\right|>c_{n}\right)\right) \\
& \quad \leq c\left(m_{0}-1\right)+c \gamma^{-p} \sum_{n=m_{0}}^{\infty} n^{-p}\left(\alpha\left(\log c_{n}\right)\right)^{p} E\left|X_{n}\right|{ }^{p} I\left(\left|X_{n}\right| \leq c_{n}\right)+O(1) \\
& \quad \leq c\left(m_{0}-1\right)+c \gamma^{-p} \sum_{n=m_{0}}^{\infty} n^{-p} E\left|X_{n} \alpha\left(\log ^{+}\left|X_{n}\right|\right)\right|^{p} I\left(\left|X_{n}\right| \leq c_{n}\right)+O(1)<\infty
\end{aligned}
$$

By Corollary 2.2, we have $b_{n}^{-1} \sum_{i=1}^{n} a_{i}\left(X_{i}^{\left(c_{i}\right)}-E X_{i}^{\left(c_{i}\right)}\right) \rightarrow 0$ a.s. The proof is complete.

From Lemma 1.4 and Theorem 2.9 we can get the following corollary.

Corollary 2.10. Let the conditions of Theorem 2.9 be fulfilled and $E X_{n}=0, n \geq 1$, and $\sum_{n=1}^{\infty} \int_{1}^{\infty} P\left(\left|X_{n}\right|>s c_{n}\right) d s<\infty$. Then $b_{n}^{-1} \sum_{i=1}^{n} a_{i} X_{i} \rightarrow 0$ a.s. 
Remark 2.11. Since an independent random variable sequence is a special NA sequence, Theorems 2.1, 2.3, 2.7, 2.8, and 2.9 all hold true for independent random variable sequences. So Theorems 2.1, 2.3, 2.7, 2.8, and 2.9 are extensions of corresponding results of Petrov [9, Chapter IX], Jamison et al. [4], Chow and Theicher [2], and Heyde [3].

\section{Acknowledgment}

This work was supported by the National Natural Science Foundation of China (Grant no. 10071058). The author thanks the referee for helpful comments.

\section{References}

[1] A. D. Barbour, L. Holst, and S. Janson, Poisson Approximation, Oxford Studies in Probability, vol. 2, The Clarendon Press; Oxford University Press, New York, 1992.

[2] Y. S. Chow and H. Teicher, Almost certain summability of independent, identically distributed random variables, Ann. Math. Statist. 42 (1971), 401-404.

[3] C. C. Heyde, On almost sure convergence for sums of independent random variables., Sankhyā Ser. A 30 (1968), 353-358.

[4] B. Jamison, S. Orey, and W. Pruitt, Convergence of weighted averages of independent random variables, Z. Wahrscheinlichkeitstheorie und Verw. Gebiete 4 (1965), 40-44.

[5] K. Joag-Dev and F. Proschan, Negative association of random variables, with applications, Ann. Statist. 11 (1983), no. 1, 286-295.

[6] J. Liu, S. Gan, and P. Chen, The Hájeck-Rènyi inequality for the NA random variables and its application, Statist. Probab. Lett. 43 (1999), no. 1, 99-105.

[7] P. Matuła, A note on the almost sure convergence of sums of negatively dependent random variables, Statist. Probab. Lett. 15 (1992), no. 3, 209-213.

[8] C. M. Newman, Asymptotic independence and limit theorems for positively and negatively dependent random variables, Inequalities in Statistics and Probability (Lincoln, Neb., 1982) (Y. L. Tong, ed.), IMS Lecture Notes Monogr. Ser., vol. 5, Inst. Math. Statist., California, 1984, pp. $127-140$.

[9] V. V. Petrov, Sums of Independent Random Variables, Ergebnisse der Mathematik und ihrer Grenzgebiete, vol. 82, Springer, New York, 1975.

[10] Q.-M. Shao, A comparison theorem on moment inequalities between negatively associated and independent random variables, J. Theoret. Probab. 13 (2000), no. 2, 343-356.

[11] Q.-M. Shao and C. Su, The law of the iterated logarithm for negatively associated random variables, Stochastic Process. Appl. 83 (1999), no. 1, 139-148.

[12] C. Su, T. Jiang, Q. H. Tang, and H. Y. Liang, The safety of negatively associated dependence structure, Chinese J. Appl. Probab. Statist. 18 (2002), no. 4, 400-404.

Gan Shixin: School of Mathematics and Statistics, Wuhan University, Wuhan 430072, China

E-mail address: shixingan@sina.com 


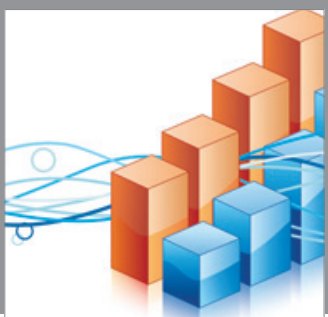

Advances in

Operations Research

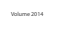

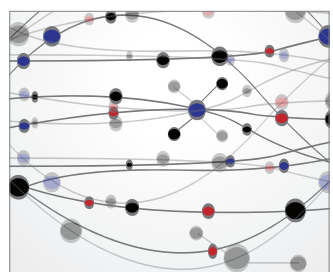

\section{The Scientific} World Journal
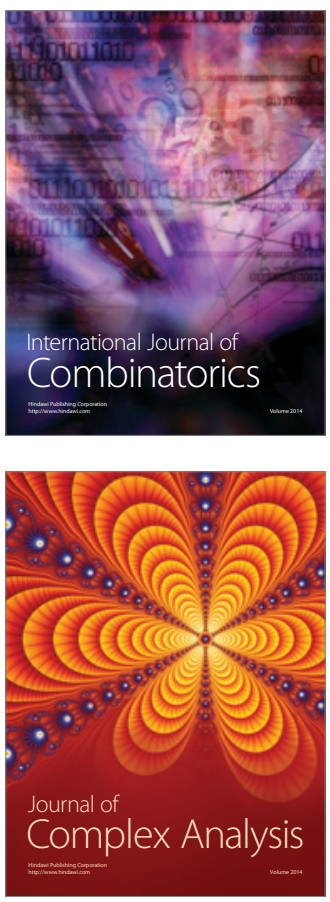

International Journal of

Mathematics and

Mathematical

Sciences
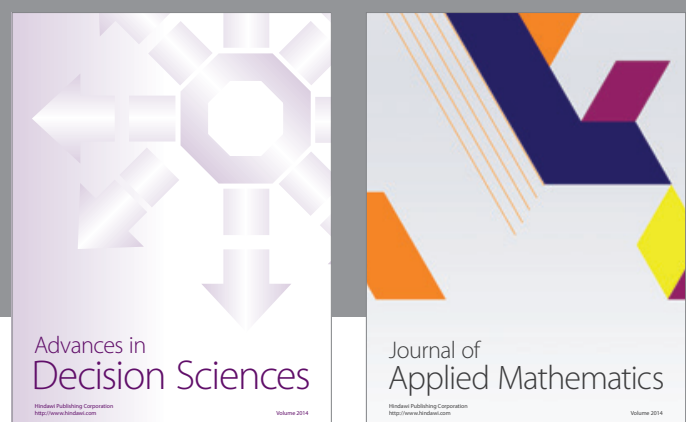

Journal of

Applied Mathematics
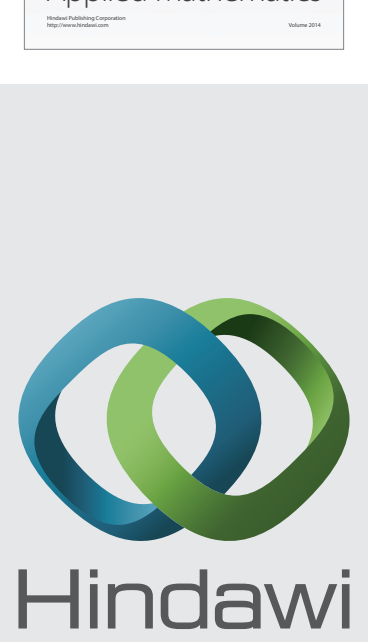

Submit your manuscripts at http://www.hindawi.com
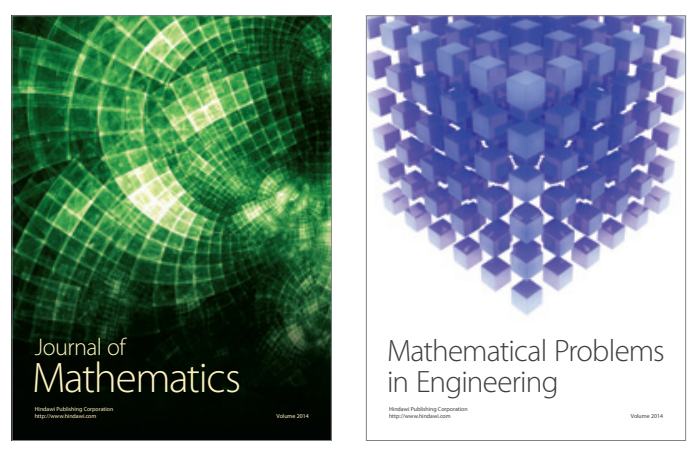

Mathematical Problems in Engineering
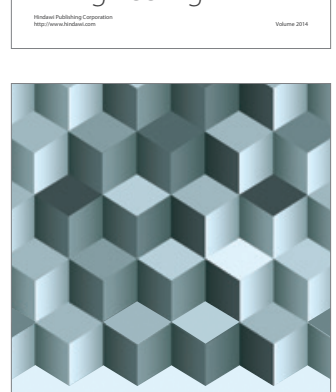

Journal of

Function Spaces
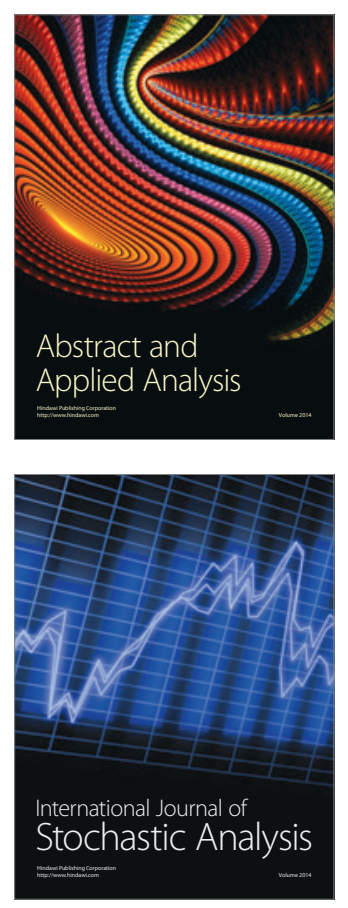

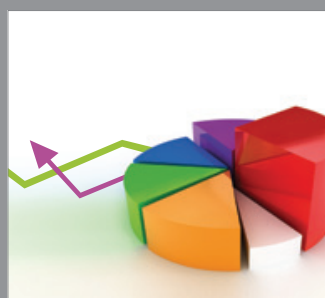

ournal of

Probability and Statistics

Promensencen
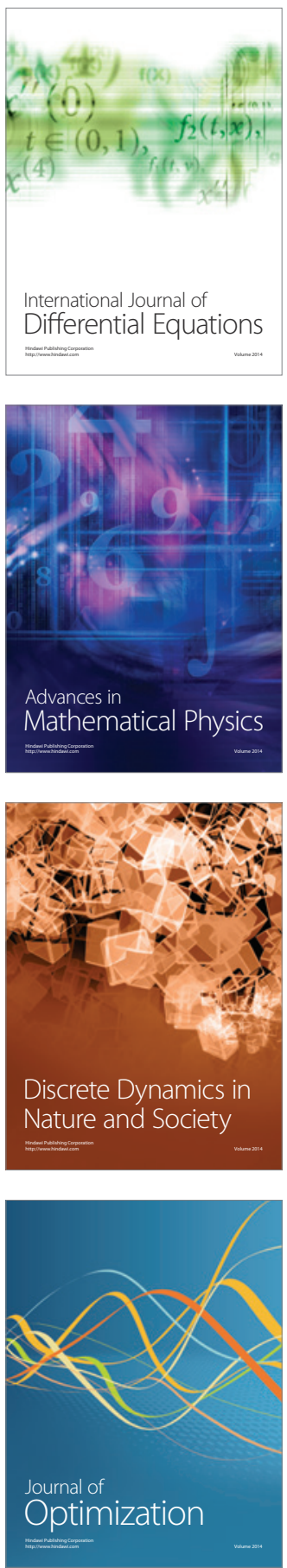